\title{
Light and Macular Degeneration: A Biophysical and Clinical Perspective
}

\author{
MARTIN A. MAINSTER \\ Kansas City, Kansas
}

\begin{abstract}
Summary
The evidence linking photic retinopathy to ageing macular degeneration (AMD) is compelling but circumstantial. The biophysical foundations of ageing theory are presented, in addition to an analysis of retinal senescence and the potential contributory role of photochemical retinal damage. Although there is pressure to implement clinical therapy for AMD based on laboratory studies of photic retinopathy, there is no evidence at this time that any such therapy is effective. Nonetheless, until the relationship between photic retinopathy and AMD is better understood, it is appropriate for individuals to use ultraviolet and deep blue protective sunglasses in bright environments, particularly if they have reduced ocular pigmentation or if they are aphakes or pseudophakes without an ultraviolet-protective intraocular lens.
\end{abstract}

\section{Time and ageing}

Notions of time are implicit in all studies of ageing. In contemporary physics, time is merely another dimension, and there are no privileged moments such as 'now'. Confusion arises because relativity theory offers convincing evidence that the magnitude of a time interval depends on the velocity and location (local space-time curvature) of the observer. Thus, the 'now' of two different observers may proceed at different rates, and the 'nows' of a group of humans are readily desynchronised by differences in their velocities and locations. In other words, 'now' may not extend beyond 'here'.

Physics does not provide an explanation for the subjective concepts of 'now' and the 'flow of time', but it does address the issue of the direction of time's arrow. ${ }^{1}$ Although useful theories have been developed to explain submicroscopic phenomena in terms of electrons moving backwards in time, when we deal with phenomena involving large numbers of particles, entropy offers a direction for time's arrow. Entropy is a measure of a system's disorder, and it either remains constant or increases in any closed system. Entropy provides only temporal asymmetry. It does not tell how rapidly 'now' moves into the future, only that the universe appears differently when viewed forward or backward in time.

All living systems are open systems that can exchange matter and energy with their surroundings. Since living systems are open, entropy can decrease temporarily during life, as manifested by the highly improbable ordered structure of living systems. The dynamic order of living systems probably arises because of self-organising fluctuations in these open, far-from-equilibrium systems ('dissipative' systems). ${ }^{2,3}$ The laser is a familiar example of a dissipative system in which there

This research was supported in part by Research to Prevent Blindness, Inc and Kansas Lions Sight Foundation, Inc.

Correspondence to: Martin A. Mainster, M.D., Ph.D., Department of Ophthalmology, Kansas University Medical Center, 39th and Rainbow Boulevard, Kansas City, Kansas 66103. 
is self-organisation of coherent emitters. The photoreceptor and retinal pigment epithelium (RPE) cell are further examples of dynamic, self-organising, open, non-equilibrium, space-time structures that arise, and after a variable period of time, decay. The openness of cellular systems is the basis of life and death. It is also the basis for our hopes to find methods for increasing the time interval in which humans and their retinae function properly.

\section{Retinal Senescence}

After development, organisms and their components deteriorate structurally and functionally. There is a remarkable similarity in the rate at which organ systems decline, however, prompting numerous vain attempts to find a single unified 'cause' for ageing. Over the years, causes proposed for ageing have paralleled areas of contemporary research interest, including toxic products of intestinal bacteria, hormone deficiencies, dehydration of body colloids, latent viruses or lethal genes, and free radical toxicity. Adding to the complexity of the problem are recent statistical studies showing that psychiatric factors influence rates of ageing and death. ${ }^{4}$ Bell's interconnectedness theorem adds further complexity, establishing non-local reality at an even more fundamental level, and forcing us to choose either objectivity (i.e. a world that exists in a definite state apart from measurement) or local causality (i.e. systems can be influenced only by mediated interactions travelling no faster than the speed of light). ${ }^{5}$

While we have far to go in our search for a unified theory of ageing, the retina does not exist independently of its host body, and it is unlikely that the problem of retinal ageing will be solved by studies limited to the retina. Nonetheless, the retina does have unique characteristics, and the identification and modification of deleterious influences may permit prolongation of retinal function. Light is one such influence. Its effects are manifested primarily at the level of the photoreceptor, RPE and Bruch's membrane.

Photoreceptor outer segment structure is a delicate balance between disc production at the proximal end of the outer segment and periodic shedding of packets of discs at the distal end of the outer segment (the photorecep-
tor-RPE juncture). ${ }^{6,7}$ Constant light blocks disc shedding but maximises disc production, resulting in longer rod outer segments. Shortened light periods and reduced temperature reduce disc production, resulting in shorter rod outer segments. Rod and cone disc shedding occurs after light onset and offset, respectively. Disc shedding and replacement provide an effective method of eliminating outer segment damage caused by light or other detrimental influences, but place a metabolic load on adjacent RPE cells responsible for phagocytosis and degradation of the phagosomes. In the aged retina, lengthy, convoluted rod outer segments are observed, suggesting impaired disc shedding, possibly because of diminished RPE function. ${ }^{8}$

The RPE is also a delicate balance, between ingestion of outer segment tips apically and apoptosis of RPE cytoplasm basally. ${ }^{9}$ Since the RPE is subject to the damaging effects of lipid oxidation in an oxygen-rich environment, it has evolved a variety of defense mechanisms including anti-oxidants such as superoxide dismutase, peroxidase, catalase and vitamin E. In addition, light absorption by melanin helps defend against high light levels, and melanin acts as an electron transfer agent. The RPE and sensory retina are also protected by macular xanthophyll. ${ }^{10}$ In senescence, there is an increase of RPE melanolipofuscin, melanolysosomes and lipofuscin, ${ }^{11}$ and a decrease in RPE melanin ${ }^{11}$ and probably other defense mechanisms. Surprisingly, the greatest increase in lipofuscin occurs between the first and second decades of life (rather than in old age), with the melanin to lipofuscin ratio reversing from $2: 1$ to $1: 2$ by the end of the second decade. ${ }^{11}$ One may speculate that the continuing increase of lipofuscin and other debris leads to a decrease in functional cytoplasmic space and progressive failure of transport, phagocytic and other metabolic functions of the ageing RPE cell.

Ageing of Bruch's membrane is due in part to deposition of RPE cytoplasmic debris, resulting in basal laminar deposits above the RPE basement membrane, and drusen on or within the inner collagenous layer..$^{9,12}$ In addition, there is mineralisation of the inner collagenous and elastic layers. It is probable though not currently proven that most RPE 
cytoplasmic debris passes through Bruch's membrane to the choroid. This is consistent with the observation that cytoplasmic debris is often absent from the inner collagenous layer underlying drusen, suggesting that drusen may result from blockage of the transport of debris through the inner collagenous layer to the choroid. ${ }^{9}$

As the retina ages, retinal thickness decreases, probably in part from a loss of retinal neurons and photoreceptors. Drusen are the earliest ophthalmoscopic sign of ageing macular degeneration (AMD). They can remain stationary, coalesce, or lead to large geographic areas of atrophic RPE. Serous drusen are often harbingers of exudative AMD, which is present in only 10 per cent of patients with AMD, but responsible for 90 per cent of all serious visual loss due to AMD ${ }^{13}$ In exudative AMD, choroidal neovascularisation may cause haemorrhagic detachment of the RPE and/or sensory retina, producing a disciform macular scar and concomitant scotoma.

Exudative AMD is rare in Blacks and Japanese,$^{14}$ consistent with the observation that increased ocular pigmentation decreases the risk of AMD in white patients. ${ }^{15}$ In addition to decreased pigmentation, dermal elastotic degeneration in sun-exposed or sun-protected tissues is another risk factor for AMD, possibly because elastic fibres in these patients have an increased susceptibility to photic or other degenerative stimuli, and the elastic layer of Bruch's membrane would be a less effective barrier to choroidal neovascularisation. ${ }^{16}$ Choroidal neovascularisation in areas of angioid streaks is common in conditions involving elastic tissue abnormalities, such as pseudoxanthoma elasticum and EhlersDanlos syndrome. On the other hand, choroidal neovascularisation is uncommon in patients with diabetic retinopathy or macular holes. Choroidal neovascularisation may also play a role in geographic AMD, with some RPE atrophy caused by cumulative, abortive, primarily subclinical leakage.

During evolution, stable systems equilibrate and become one with the environment; unstable systems degrade and perish; and only systems of a oscillatory nature survive. ${ }^{2}$ Evolution has been fuelled by solar electromagnetic radiation which varies periodically in intensity as the earth spins on its axis and orbits the sun. Complex systems operate by periodic transformation of energy into work, and many biological systems exhibit a circadian rhythm that is largely independent of temperature and environmental influences.

The photoreceptor and RPE cell are complex open systems, incorporating autocatalytic chemical reactions, undergoing auto-oscillations in structure and function, dependent on the provision of essential raw materials and the removal of cellular debris, and existing in a dynamic order subject to numerous local catastrophes. This complex, dynamic order eventually collapses in senescence. While we readily appreciate changes at individual points in time (such as lipofuscin accumulation), or those occurring during short intervals of our life (such as choroidal neovascularisation), we may fail to observe very slowly evolving dynamic changes equally fundamental to our understanding of senescence.

\section{Photic Retinopathy}

In 1900, Max Plank deduced the continuous spectrum of light emitted by a perfect absorber ('blackbody') by assuming that the light is produced in discrete packets, now known as photons, and that the energy of each photon is inversely proportional to its wavelength. Thus, the energy of an ultraviolet light photon is greater than that of blue light photon, and a photon of blue light has greater energy than a green light photon. If a photon has sufficient energy, it can produce a change in the energy state of an atom or molecule, inducing conformational changes, or even promoting chemical reactions known as photochemical reactions. The yellowing of transparent polymethylmethacrylate in sunlight and photic retinopathy are both photochemical effects.

Photochemical retinal damage was first demonstrated in rats, and subsequently in primates and humans. ${ }^{17-21}$ Photic retinopathy occurs at temperature increases far below the 10 to 20 degrees $C$ temperature rise needed for photocoagulation. Solar retinitis (eclipse retinopathy) is an example of photic retinopathy, since unaided solar observation produces a temperature increase of less than 4 degrees $C .{ }^{22}$ Photochemical damage can affect the photo- 
receptors, the RPE, or both the neurosensory retina and RPE. ${ }^{17-20}$

Photochemical damage may be divided into two categories. ${ }^{17}$ At the lowest light levels (class 1), photic retinopathy appears to be mediated by visual photopigments, as in rat experiments ${ }^{17}$ and later primate cone damage studies. ${ }^{20}$ At higher light levels (class 2 ) that are still below photocoagulation irradiances, photic retinopathy is probably an actinic process, but the identity of target molecules in the RPE and neurosensory retina is currently unknown. Solar retinitis and welder's maculopathy most likely are class 2 effects, not primarily of photopigment origin. A striking feature of class 2 photic retinopathy is that damage susceptibility increases with decreasing wavelength, so that ultraviolet radiation and blue light pose the greatest risk. ${ }^{18-20}$ This latter finding is consistent with the fact that photon energy increases with decreasing wavelength, so there is a higher probability that a shorter wavelength photon will have sufficient energy to induce a critical reaction. Class 2 photic retinopathy appears to be additive, and is enhanced by increased oxygen tension. ${ }^{23,24}$ It depends on the species, and the location and extent of the exposed retina. It also depends on the duration, intensity and spectrum of the light source.

Photic retinopathy in primates may occur because the retina's defence mechanisms against toxic free radicals from light and oxygen are overwhelmed by light levels exceeding those normally encountered in our environment. While a free radical mechanism gains considerable support from studies showing that susceptibility to photic retinopathy increases with increased blood oxygen tension, ${ }^{23.24}$ other damage mechanisms may be involved. For example, light could damage critical intracellular biochemical systems, responsible for controlling ionic homeostasis, calcium storage, transport functions, cell respiration, or RPE phagocytosis. In addition, maintenance of photoreceptor discs in a bleached state for a prolonged interval of time could lead to thermal instabilities that ultimately disrupt the dynamic structure of the outer segment. ${ }^{25}$ Photic retinopathy probably comprises a continuum of related hazards, the nature of biochemical events being deter- mined by the exposure history and the effectiveness of individual retinal defence mechanisms.

There has been a marked increase in reported cases of photic retinopathy from operating microscopes in the past few years, complementing previous reports of photochemical retinal damage from solar and welding arc observation, and emphasising the clinical significance of photic retinopathy. ${ }^{26}$ Unfortunately, experimental studies show that mild photochemical retinal damage may not be visible ophthalmoscopically, so clinical reports probably represent only the most severe injuries. It is also possible that intense erythropsia leaves permanent effects that are not initially apparent, at least ophthalmoscopically, and that this condition may be the clinical analog of blue-sensitive cone photochemical damage observed in primates at very low light levels.

The electromagnetic spectrum spans a continuous range of wavelengths, including visible light between 400 and $700 \mathrm{~nm}$, and ultraviolet radiation at shorter wavelengths. In a normal human eye, the cornea is opaque to ultraviolet light below $300 \mathrm{~nm}$, so the cornea shields internal ocular components from potential harm from light below $300 \mathrm{~nm} .{ }^{27}$ In the near-ultraviolet region between 300 and $400 \mathrm{~nm}$, however, the cornea is transparent. Fortunately, the crystalline lens absorbs near-ultraviolet radiation between 300 and $400 \mathrm{~nm},{ }^{27}$ thereby shielding the retina from potential harm. When the crystalline lens is removed in cataract surgery, however, the retina's near-ultraviolet light protection is also removed. Replacing the crystalline lens with a clear PMMA intraocular lens provides little protection, because clear PMMA allows most of the near-ultraviolet light to get through to the retina. ${ }^{28,29}$ Use of a UV-absorbing IOL increases this protection, but there is considerable variability in the protection afforded by commercially available lenses, and none provides the blue light protection offered by the native crystalline lens.

\section{Photic Retinopathy and Macular Degeneration}

The evidence linking photic retinopathy to macular degeneration is circumstantial but 
compelling, and cumulative photochemical retinal damage from environmental light exposure has been proposed as a cause for AMD. ${ }^{29}$ Indeed, ultraviolet radiation was implicated in macular degeneration over sixty years ago, long before solar retinitis was known to be photochemical in origin. ${ }^{30}$ The primary reason initially for linking UV radiation to macular degeneration was the clinical observation in a large series of patients that macular degeneration was less common in patients with cataract formation..$^{30}$ This inverse relationship is supported by recent statistical studies showing a reduced incidence of macular degeneration in patients with nuclear (but not cortical) cataract formation, ${ }^{31}$ and a threefold increase in the incidence of drusen formation in the non-phakic eye (aphakic or clear PMMA IOL) of patients who have undergone unilateral cataract extraction..$^{32}$

In addition to these statistical studies, and the similarity of retinal findings in photic retinopathy and macular degeneration, further evidence linking these two disorders arose in 1941-4 and 1966-9..$^{33}$ Aside from pilots, antiaircraft observers and others who might be exposed to solar retinitis in their line of duty, a similar condition known as foveomacular retinitis arose in personnel without a history of sun gazing who were stationed generally in bright environments. Macular disorders were even more prevalent among prisoners of war in the south Pacific, where malnutrition was combined with high environmental illuminances and temperatures. ${ }^{34}$

\section{Clinical Implications}

While we are only beginning to understand the nature of photic retinopathy and its relation to macular degeneration, AMD is a widespread problem, and there is pressure to translate experimental results from photic retinopathy studies into clinical therapies. There is no proof at this time that any therapy reduces the risk of AMD, and patients should not be misled by speculative extrapolation of laboratory data on photic maculopathy.

Since humans and experimental animals develop retinal abnormalities similar to macular degeneration when deprived of key vitamins and cofactors, one therapy proposed for macular degeneration is dietary sup- plementation of antioxidant vitamins $\mathrm{E}$ and $\mathrm{C}$, and cofactors selenium (for glutathione peroxidase) and zinc (for catalase). While deficiencies of antioxidants and cofactors may increase susceptibility to AMD and photic retinopathy, there is no evidence that supplements help individuals already receiving a balanced diet. Although laboratory studies show that vitamin Csupplementation protects rats against photic retinopathy, ${ }^{35}$ clinical studies show no correlation between exudative macular degeneration and blood levels of vitamins A, C or E. ${ }^{16}$ In fairness, however, since the largest increase in lipofuscin occurs between the first and second decade, ${ }^{11}$ it may be necessary to correlate blood levels in youth to identify individuals at subsequent risk to AMD.

Bright, warm environments are conducive to foveomacular retinitis, at least in the military, but the aetiology of this disorder remains puzzling, along with why it is sporadic and apparently absent in civilian populations exposed to similar climates. Solar retinopathy is a potential contributing factor, but there is no firm evidence for this association, and the many other possible explanations include immunisations, chorioretinal infections, liver diseases, drug photosensitisation, and vitamin deficiencies. In any event, malnutrition hastens macular degenerative changes in white people in bright, hot environments, suggesting the need for a better understanding of the role of light and ambient temperature in premature macular ageing, and the value of light protection in patients with malabsorption syndromes.

One effective form of light protection is sunglasses which screen out UV-radiation and blue light below $450 \mathrm{~nm}$. These sunglasses should be used in bright environments by aphakes or clear PMMA (i.e. PMMA without UV-absorbing chromophores) pseudophakes whose retinae are no longer protected by the crystalline lens. ${ }^{28,36}$ Whether such sunglasses provide any significant protection for phakic individuals with normal nutrition in temperate climates remains to be determined. In any event, just as it is important to wear protective ear plugs around intense sound sources such as jet aircraft, and use sunscreen lotion in high ultraviolet radiation environments, it also makes sense to wear appropriate protective 
sunglasses in bright environments, at least until the role of photic retinopathy in macular degeneration is better understood. It must be emphasised that most sunglasses are poorly labelled, however, and that poor sunglasses are potentially more harmful than none at all. For example, a sunglass that filters out most of the visible spectrum but transmits UV-radiation and deep blue light would relax the eye's squint and pupillary constriction defence mechanisms, thereby placing the user at greater risk to lenticular or retinal damage. ${ }^{37}$

Clinical light sources must provide adequate light levels for both clear and hazy media, and levels needed for patients with hazy media may be uncomfortable or dangerous for patients with clear media. Several methods are available for improving the safety of clinical examinations. (1) Filtering out light with wavelengths below $450 \mathrm{~nm}$ would reduce the risk of photic retinopathy without significantly impairing visualisation of necessary clinical detail. Filtering out wavelengths greater than $700 \mathrm{~nm}$ would eliminate needless infrared radiation which could thermally-enhance photochemical damage.$^{36}$ (2) Examinations should always be performed with a minimum amount of light exposure for both patient comfort and safety. This fact should be understood by residents mastering diagnostic and surgical skills. ${ }^{36}$ (3) Occlusion devices should be used whenever fundus visualisation is unnecessary, whether by occluding part of the illuminating beam or placing an opaque shield on the cornea. In this latter case, highly absorbing ( $v s$. absorbing and scattering) occluders such as those made from black PMMA should be avoided since they can attain temperatures high enough to cause thermal keratitis.

Ophthalmologists themselves are at risk to photic retinopathy from incandescent or laser light scattered back at them off optical interfaces such as contact examination lens surfaces and mirrors. A primary area of concern is photocoagulation with visible light. The scattered light viewed when observing a photocoagulator aiming beam usually is not attenuated by protective filters. Clinicians are subject to periodic dazzling reflexes that are safe in the short term, but have an unknown, cumulative, long-term effect. Thus, it is probably safer to use dye or krypton laser yellow or red light for routine photocoagulation since these have a lower potential for producing. photic retinopathy in the operator or patient.

Light is the source of life and the origin of decay. It is responsible for retinal development and involution. The photoreceptor, RPE and Bruch's membrane are all complex, open systems in dynamic order. Excessive light disrupts that order, but some light is undoubtedly needed to stimulate retinal defences against light damage, and cyclic light is required to entrain the rhythm of structure and function of the outer segment, RPE and Bruch's membrane. Light surely plays a role in retinal senescence, just as usage is related to the longevity of any implement. It is not known, however, if light exposure history is any more or less significant over a lifetime than a myriad of other factors such as climactic temperature, basal body temperature, childhood febrile illnesses, and variations or interruptions in the light/dark cycle (e.g. by leaving room lights on constantly in premature infant nurseries, or by time zone changes in transcontinental travel).

The patient with advanced glaucoma has a narrow perspective of space, but we have a narrow perspective of time, viewing events with a patience proportional to the longevity of our species. Contemporary scientific methods are not well-suited to studying events, such as 'human ageing, whose duration is comparable to an investigator's lifespan. Indeed, the 'progress' to be demonstrated in a contemporary research grant limits techniques to those which show 'results' within a 2 or 3 year cycle of research funding. Just as we fail to discern motion in the subtle unfolding of a rose, we may fail to appreciate the dynamics of slowlydeveloping intraretinal changes, or detect improbable events such as electron tunnelling whose repetitive effects could be significant over a lifetime. Unfortunately, the vital mathematical methods needed to understand these long-term processes, such as non-equilibrium thermodynamics and catastrophe theory, are abstruse disciplines in the early stages of their development. Light undoubtedly affects retinal senescence, but intraretinal dynamics and ageing in a healthy human are too complex to expect serendipitous cures, and the solution of retinal ageing awaits better comprehension of 
human ageing and relevant interactions in nature.

\section{References}

${ }^{1}$ Morris R: Time's arrows: scientific attitudes toward time. New York: Simon and Schuster, 1985; 7-27.

2 Volkenshtein MV: Biophysics. Moscow: Mir, 1983; 339-45.

${ }^{3}$ Prigogine I, Stengers I: Order out of chaos. New York: Bantam, 1984; 189-209.

${ }^{4}$ Dossey L: Space, time and medicine. Boston: New Science Library, 1982; 59-71.

${ }^{5}$ Herbert N: Quantum reality. Garden City NY: Anchor, 1985; 211-31.

6 Young RW: Biological renewal. Applications to the eye. Trans. Opthalmol. Soc. UK. 1982; 102: 42-75.

${ }^{7}$ Besharse JC: Photosensitive membrane turnover: differentiated membrane domains and cell-cell interaction. In: Adler R, Farber D, eds. The retina: a model for cell biology studies, Part I. Orlando: Academic Press, 1986; 297-352.

${ }^{8}$ Marshall J, Grindle J, Ansell PL, Borwein B: Convolution in human rods: an ageing process. $\mathrm{Br}$. J. Ophthalmol. 1979; 63: 181-7.

${ }^{9}$ Feeney-Burns L, Ellersieck MR: Age-related changes in the ultrastructure of Bruch's membrane. Am. J. Ophthalmol. 1985; 100: 686-97.

${ }^{10}$ Snodderly DM, Auran JD, Delori FC: The macular pigment. II. Spatial distribution in primate retinas. Invest. Ophthalmol. Vis. Sci. 1984; 25: 67485.

${ }^{11}$ Feeney-Burns L, Hilderbrand ES, Eldridge S: Ageing human RPE: morphometric analysis of macular, equatorial and peripheral cells. Invest. Ophthalmol. Vis. Sci. 1984; 25: 195-200.

12 Sarks SH: Drusen and their relationship to senile macular degeneration. Austral. J. Ophthalmol. 1980; 8: 117-30.

${ }^{13}$ Ferris FL, Fine SL, Hyman L: Age related macular degeneration and blindness due to neovascular maculopathy. Arch. Ophthalmol. 1984; 102: 1640-2.

${ }^{14}$ Hoshino M, Mizuno K, Ichikawa H: Aging alterations of retina and choroid of Japanese: light microscopic study of macular region of 176 eyes. Jpn. J. Ophthalmol. 1984; 28: 89-102.

${ }^{15}$ Weiter JJ, Delori FC, Wing GL, Fitch KA: Relationship of senile macular degeneration to ocular pigmentation. Am. J. Ophthalmol. 1985; 99: 185-7.

${ }^{16}$ Blumenkranz MS, Russell SR, Robey MG, KottBlumenkranz R, Penneys N: Risk factors in agerelated maculopathy complicated by chloroidal neovascularisation. Ophthalmol. 1986;93: 552-7.

${ }^{17}$ Noell WK: Possible mechanisms of photoreceptor damage by light in mammalian eyes. Vision Res. 1980; 20: 1163-71.

${ }^{18}$ Ham WT, Mueller HA, Sliney DH: Retinal sensitivity to damage from short wavelength light. Nature. 1976; 260: 153-5.

${ }^{19}$ Lawwill T: Three major pathologic processes caused by light in the primate retina: a search for mechanisms. Trans. Am. Ophthalmol. Soc. 1982; 80: 517-79.

${ }^{20}$ Sperling HG, Johnson C, Harwerth RS: Differential spectral photic damage to primate cones. Vision Res. 1980; 20: 1117-25.

${ }^{21}$ Tso MOM, LaPiana FG: The human fovea after sungazing. Trans. Am. Acad. Ophthalmol. Otolaryngol. 1975; 79: 788-95.

22 White TJ, Mainster MA, Wilson PW, Tips JH: Chorioretinal temperature increases from solar observation. Bull. Math. Biophys. 1971; 33: 1-17

${ }^{23}$ Crockett RS, Lawwill T: Invest. Ophthalmol. Vis. Sci. Supp 1983; 24: 253.

${ }^{24}$ Ruffolo JJ, Ham WT, Mueller HA, Millen JE: Photochemical lesions in the primate retina under conditions of elevated blood oxygen. Invest. Ophthalomol. Vis. Sci. 1984; 25: 893-8.

${ }^{25}$ Mainster MA: Destructive light adaptation. Ann. Ophthalomol. 1970; 2: 44-8.

${ }^{26}$ Fishman GA: Light-induced maculopathy from surgical microscopes during cataract surgery. In: Ernest JT, ed. 1985 Year Book of Ophthalmology. Chicago, Year Book Medical Publishers, 1985; 177-80.

${ }^{27}$ Boettner EA, Wolter JR: Transmission of the ocular media. Invest. Ophthalmol. Vis. Sci. 1962;1:77683.

${ }^{28}$ Mainster MA: Spectral transmittance of intraocular lenses and retinal damage from intense light sources. Am. J. Ophthalmol. 1978; 85: 167-70.

${ }^{29}$ Mainster MA: Solar retinitis, photic maculopathy and the pseudophakic eye. Am. Intra-Ocular Implant. Soc. J. 1978; 4: 84-6.

${ }^{30}$ van der Hoeve $\mathrm{J}$ : Eye lesions produced by light rich in ultraviolet rays. Senile cataract senile degeneration of the macula. Am. J. Ophthalmol. 1920; 3: 178-94.

${ }^{31}$ Sperduto RD, Hiller R, Seigel D: Lens opacities and senile maculopathy. Arch. Ophthalmol. 1981;99: 1004-8.

${ }^{32}$ Guyer DR, Alexander MF, Auer CL, Hamill MB, Chamberlin JA, Fine SL: A comparison of the frequency and severity of macular drusen in phakic and non-phakic eyes. Invest. Ophthalmol. Vis. Sci. Supp 1986; 27: 20.

${ }^{33}$ Marlor RL, Blais BR, Preston FR, Boyden DG: Foveomacular retinitis, an important problem in military medicine: epidemiology. Invest. Ophthalmol. 1973; 12: 5-16.

${ }^{34}$ Clarke CA, Sneddon IB: Nutritional neuropathy in prisoners-of-war and internees from Hong-Kong. Lancet. 1946; 1: 734-7.

${ }^{35}$ Li ZY, Tso MOM, Wang H, Organisciak DT: Amelioration of photic injury in rat retina by ascorbic acid: a histopathologic study. Invest. Ophthalmol. Vis. Sci. 1985; 26: 1589-98.

${ }^{36}$ Mainster MA, Ham WT, Delori FC: Potential retinal hazards. Instrument and environmental light sources. Ophthalmol. 1983; 90: 927-31.

${ }^{37}$ Sliney DH: Eye protective techniques for bright light. Ophthalmol. 1983; 90: 937-44. 